\title{
How-I-Do-It
}

\section{Percutaneous trans-hepatic rescue and neo-creation of a post-surgical complete hepaticojejunostomy dehiscence}

\author{
Umberto Geremia Rossi', Andrea DeCensi ${ }^{2}$, Gian Andrea Rollandi ${ }^{3}$, Marco Filauro ${ }^{4}$ \\ 'Department of Diagnostic Imaging, Interventional Radiology Unit, E.O. Galliera Hospital, Genova, Italy, \\ ${ }^{2}$ Department of Medical Area, Medical Oncology Unit, E.O. Galliera Hospital, Genova, Italy, \\ ${ }^{3}$ Department of Diagnostic Imaging, Radiology Unit, E.O. Galliera Hospital, Genova, Italy, \\ ${ }^{4}$ Department of Abdominal Surgery, General and Hepato-Bilio-Pancreatic Surgery Unit, E.O. Galliera Hospital, Genova, Italy
}

\begin{abstract}
Biliary leakage complicating hepaticojejunostomy is a therapeutic dilemma for surgeons, gastroenterologist, and interventional radiologist. It is a major cause of postoperative morbidity. Percutaneous biliary intervention techniques have been developed for treating benign post-surgical biliary disease in patients not having severely compromised clinical conditions. Interventional radiology manoeuvres are especially indicated as the first line of treatment if endoscopic procedures are unfeasible for patients due to postsurgical anatomical modifications. We present a case of post-surgical complete hepaticojejunostomy dehiscence that was treated totally by percutaneous techniques with trans-hepatic rescue and hepaticojejunostomy neo-creation.
\end{abstract}

Key Words: Biliary; Interventional radiology; Drainage; Surgery; Liver

\section{INTRODUCTION}

Hepaticojejunostomy $(\mathrm{HJ})$ is a common surgical procedure. However, leak/dehiscence of anastomosis has an incidence ranging from $0.4 \%$ to $12 \%$ [1-3]. This clinical situation requires a rapid treatment as it is a major cause of postoperative morbidity [1]. Endoscopic and "rendez-vous" procedures are usually infeasible in these patients due to modified bowel anatomies after surgeries. Percutaneous biliary interventions have been described for the treatment of this post-surgical biliary disease [1-5]. We present a case of post-surgical complete HJ dehiscence that was treated by percutaneous techniques (drainage and bio-resorbable stenting) with trans-hepatic rescue and $\mathrm{HJ}$ neo-creation.

Received: January 23, 2021, Revised: March 8, 2021,

Accepted: March 10, 2021

Corresponding author: Umberto Geremia Rossi Department of Diagnostic Imaging, Interventional Radiology Unit, E.O. Galliera Hospital, Mura delle Cappuccine, 14, Genova 16128, Italy

Tel: +39-010-5634154, Fax: +39-010-5634154, E-mail: umberto.rossi@galliera.it ORCID: https://orcid.org/0000-0001-9785-4237

Copyright $(\mathcal{C})$ The Korean Association of Hepato-Biliary-Pancreatic Surgery This is an Open Access article distributed under the terms of the Creative Commons Attribution Non-Commercial License (http://creativecommons.org/licenses/by-nc/4.0) which on-commercial use, distribution, and reproduction in any medium, provided the origina permits unrestricted work is properly cited.

\section{CASE REPORT: TECHNIQUE}

A 48-year-old male underwent pancreatic surgery with $\mathrm{HJ}$ for a locally advanced pancreatic adenocarcinoma. On the 10th postoperative day, the patient started to have upper abdominal pain, fever, and evidence of bilious output from surgical drain. He underwent urgent multi-detector computed tomography that revealed a collection, with bubble of air at the level of $\mathrm{HJ}$ without intrahepatic biliary ducts dilatation (Fig. 1). Complete $\mathrm{HJ}$ dehiscence was suspected. It was confirmed by a percutaneous catheter contrast study (Fig. 2). His liver function values were within normal limits except for gamma-glutamyl transpeptidase of $93 \mathrm{U} / \mathrm{L}$ (range, 8-61 U/L). After a multidisciplinary discussion, the patient was a candidate for a first-line percutaneous approach. From right surgical drainage, a retrograde opacification of non-dilated intrahepatic biliary tree was achieved (Fig. 2). The opacification of the intrahepatic biliary tree allowed us to indicate the possible percutaneous manoeuvre, while knowing that the puncture of non-dilated biliary ducts could be burdened by a high risk of technical failure with possible liver complications [1]. With a Seldinger's technique, right percutaneous trans-hepatic internal-external biliary drainage ( $8 \mathrm{Fr}$ ) was inserted under fluoroscopic guidance, with its tip inserting into the anastomotic bowel loop (Fig. 3). The drainage ensured regular outflow of bile from the liver to the bowel, leading to the creation a retroperitoneal fibrosis with 


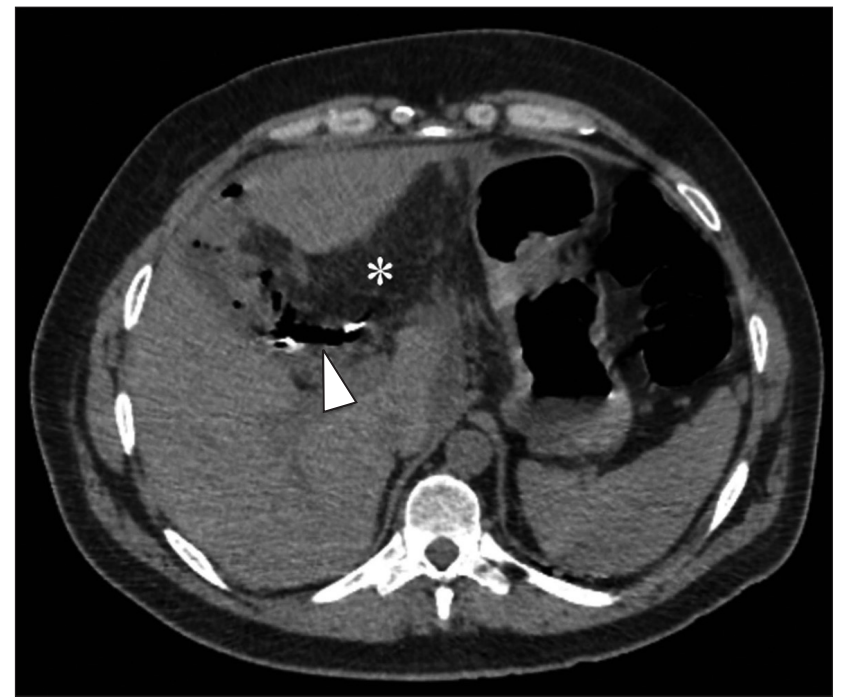

Fig. 1. Multi-detector computed tomography axial image demonstrating a perihepatic collection $\left(^{*}\right)$ with air bubbles at the level of hepaticojejunostomy (arrowhead).

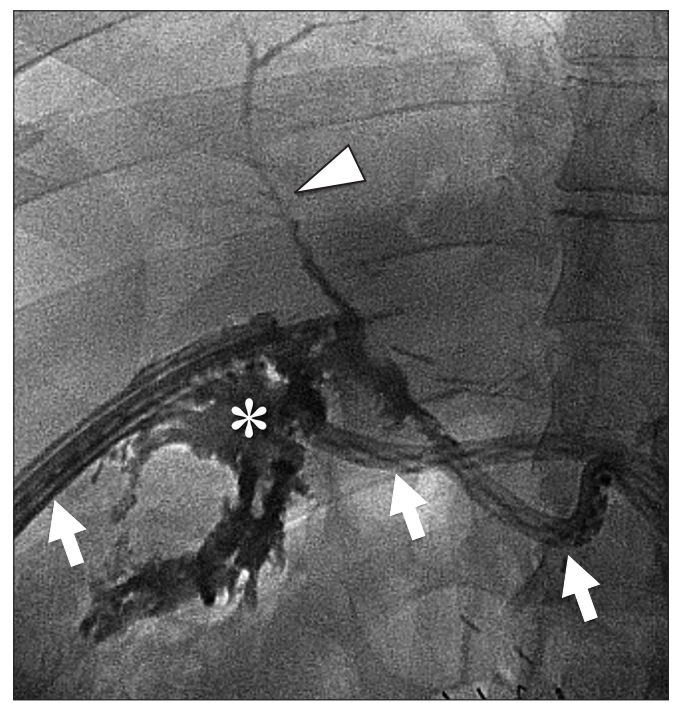

Fig. 2. Percutaneous catheter contrast study done from surgical drains (arrows) showing a collection of contrast medium $\left(^{*}\right)$ that leaks from the hepaticojejunostomy complete dehiscence. Retrograde opacification of not dilated intra-hepatic biliary was noted (arrowhead). Note that there was no opacification of the anastomotic bowel loop.

consequent a neo-HJ in one month. At three months follow-up, a HJ stenosis was noted on cholangiography (Fig. 4). Endobiliary interventional radiology approach was chosen. Given the failure of three high pressure (16 Atm) balloon bilioplasty attempts for recoiling of the stenosis, a $10 \mathrm{~mm} \times 45 \mathrm{~mm}$ bio-resorbable biliary stent (SX-ELLA DV biliary; ELLA-CS, Hradec Kralove, Czech Republic) was positioned which ensured regular patency of the neo-HJ without complication (Fig. 5). Fol-

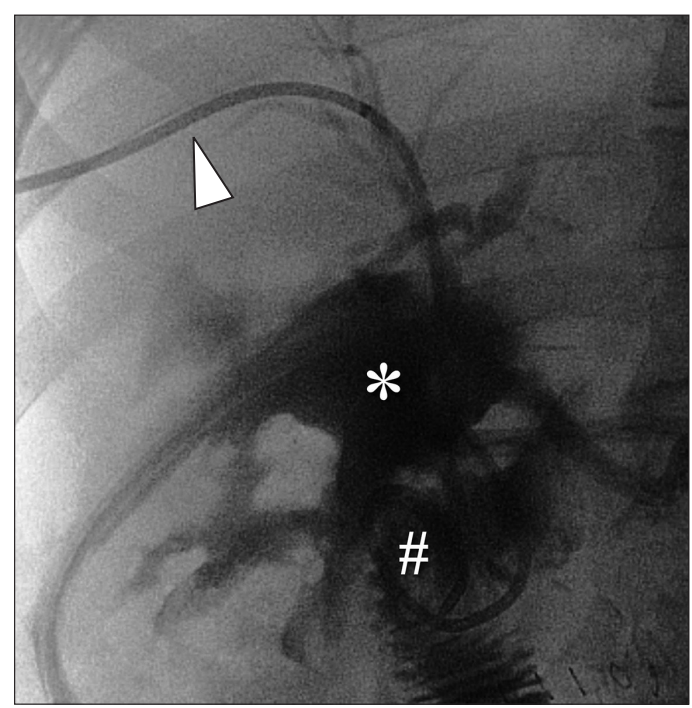

Fig. 3. Percutaneous trans-hepatic cholangiography from internalexternal biliary drainage (arrowhead) with its tip inserted into the anastomotic bowel loop (\#). Note the persistent collection of contrast medium $\left(^{*}\right)$ from the hepaticojejunostomy complete dehiscence.

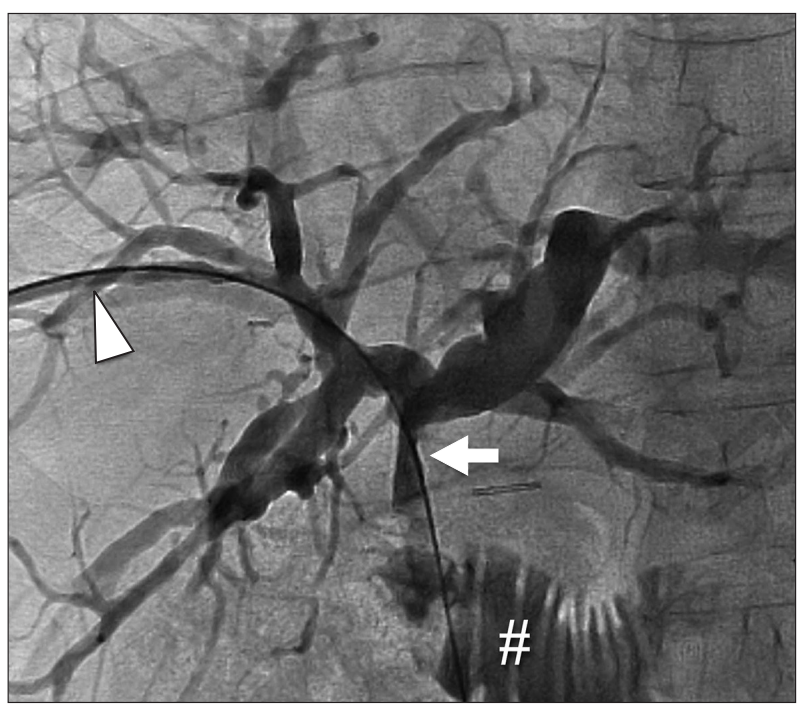

Fig. 4. Percutaneous trans-hepatic cholangiography from internalexternal biliary drainage (arrowhead) at one month with regular flow of bile from the liver to the bowel (\#). Note the absence of contrast medium collection and creation of a neo-hepaticojejunostomy that appears stenotic (arrow).

low-up studies at 32 months after stenting, including clinical observation and laboratory tests, showed no complications and regular patency of the neo-HJ (Fig. 6).

\section{DISCUSSION}

Biliary leaks remain a significant source of morbidity in patients undergoing pancreatic surgery [1-5]. The incidence of $\mathrm{HJ}$ 


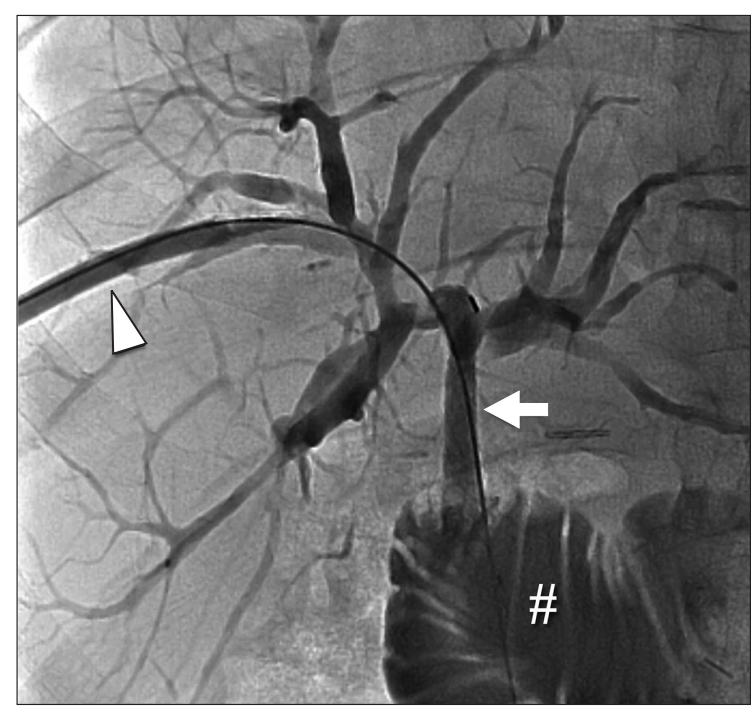

Fig. 5. Percutaneous trans-hepatic cholangiography from the external introducer (arrowhead) that shows a regular diameter of the neohepaticojejunostomy (arrow) after bioresorbable stent deployment with regular floe to the bowel (\#).

dehiscence after pancreatic open surgery ranges from $3 \%$ to $8 \%$ [1]. HJ dehiscence is generally due to bile duct ischemia with consequent necrosis leading to a fistula [2]. The most complicate biliary leak to treat is complete $\mathrm{HJ}$ dehiscence $[1,2,6]$. Clinical signs of $\mathrm{HJ}$ dehiscence may include leucocytosis, fever, abdominal pain, and presence of bile-looking from surgical drain [1-3]. This complication requires prompt diagnosis and subsequent treatment. This diagnosis can be confirmed by imaging ultrasound, computed tomography, and magnetic resonance that can identify bilomas and/or the biliary leak [1]. A multidisciplinary approach is essential in these cases to evaluate the previous pathology, post-surgical anatomy, types of biliary leakage, and possible treatments (from the less invasive one to the most invasive one). Traditionally, biliary leakages have been treated by surgery. However, endoscopic and/ or interventional radiology procedures are adopted recently. Endoscopic procedures are frequently technically unfeasible in these patients due to a modified bowel anatomy [1,2]. According to the International Study Group of Liver Surgery of 2011, for patients with a moderately compromised clinical condition, percutaneous procedures are indicated as the first line treatment [7]. Percutaneous trans-hepatic cholangiography followed by percutaneous trans-hepatic biliary drainage (PTBD) can confirm the diagnosis and treat biliary collection and fistula by leakage healing [1-3]. However, when HJ dehiscence is completed, PTBD may be technically unfeasible due to the absence of intrahepatic biliary duct dilatation, unlike an occlusive pathology [8]. This decompression of intrahepatic biliary ductal does not allow an ultrasound-guided puncture. Therefore, percutaneous transhepatic access can be done by retrograde visualization of the intrahepatic biliary tree from surgical drainage

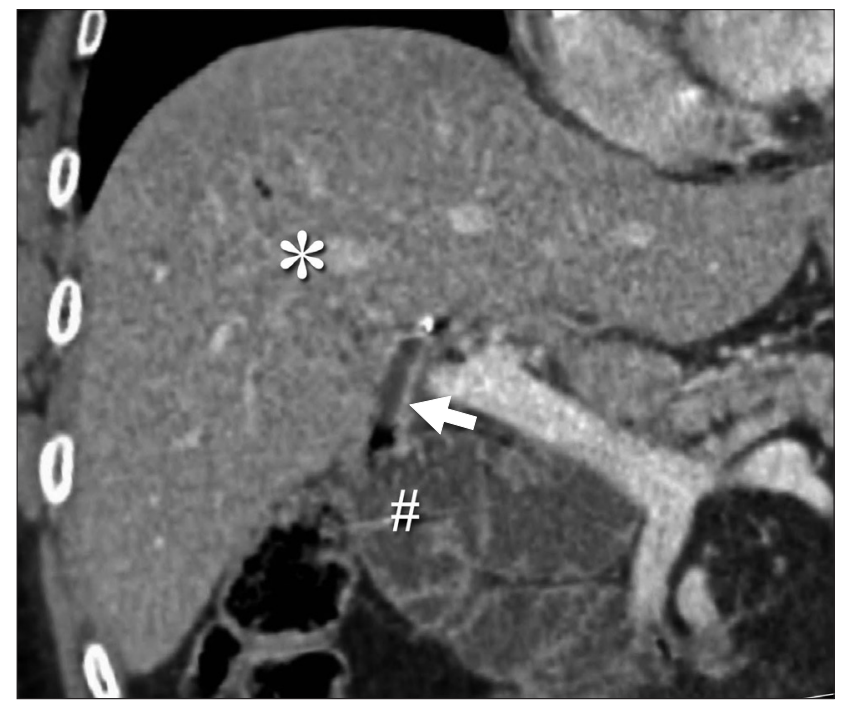

Fig. 6. Coronal computed tomography image at follow-up that confirms a regular diameter of the neo-hepaticojejunostomy (arrow) between the liver $\left(^{*}\right)$ and the bowel (\#).

or by a direct puncture of a biliary duct near the hepatic hilum with consequent contrast media filling and opacification of the intrahepatic biliary tree. Despite this technical limitation of biliary tract decompression for interventional radiological approach and possible technical unsuccessful, a percutaneous attempt can be indicated in most cases before a more invasive surgical approach [2].

Only a few authors have described successful percutaneous approach in patients with altered anatomies due to previous surgeries [1-7,9-12]. Percutaneous manoeuvres performed range from internal-external biliary drainage to stent deployment: plastic, metallic, and bioresorbable.

Percutaneous internal-external biliary drainage is the firstline manoeuvre that allows the drainage of the bile (both internally and externally) with the aim to detain and seal the biliary leak. Timing of this approach is directly proportional to the extent of the anastomotic dehiscence. Thus, the resolution period with percutaneous treatment of $\mathrm{HJ}$ dehiscence is highly variable, with a minimum time of about 30 days (as in our case) and an average of 180 days [1-3]. Cholangiography is the diagnostic imaging exam that can reveal successful healing of HJ. However, if no signs of anatomical anastomotic and clinical improvement are shown during follow-up controls, surgical intervention is mandatory. Antibiotic therapy and daily trans-catheter saline solution syringe injection $(10-20 \mathrm{~mL})$ are necessary to reduce the risk of infection and occlusion of the catheter itself [13].

Plastic stent has the advantage of possible removal. However, their small diameters (maximum up to $14 \mathrm{Fr}$ ) give them the possible risk of occlusion, especially in case of biliary sludge $[1,2,5]$. Metallic bare and covered stents are effective but ir- 
reversible solutions. Over time, metallic stents can produce a "foreign body" effect with fibrosis that may need or complicate a possible percutaneous or surgical re-intervention $[5,9,11]$. Bio-resorbable stents have advantages of both plastic and metal devices. With a large diameter (up to $10 \mathrm{~mm}$ ), they allow a long-term dilatation without needing additional manoeuvres. Stent degradation occurs by hydrolysis within six months, without needing its removal or the risk of a "foreign body" effect $[9,11]$.

In conclusion, complete $\mathrm{HJ}$ dehiscence is a severe and critical complication of open surgery. In selected cases of HJ dehiscence, percutaneous interventional radiological manoeuvres are even more important than ever. A percutaneous approach can solve this post-surgical complication with minimally invasive techniques using drainage and stents.

\section{CONFLICT OF INTEREST}

No potential conflict of interest relevant to this article was reported.

\section{ORCID}

Umberto Geremia Rossi, https://orcid.org/0000-0001-9785-4237

Andrea DeCensi, https://orcid.org/0000-0003-2635-4491

Gian Andrea Rollandi, https://orcid.org/0000-0002-3786-7658

Marco Filauro, https://orcid.org/0000-0003-4029-400X

\section{AUTHOR CONTRIBUTIONS}

Conceptualization: UGR. Data curation: All authors. Methodology: UGR, MF. Visualization: UGR, GAR. Writing - original draft: UGR, AD. Writing - review \& editing: All authors.

\section{REFERENCES}

1. Mansueto G, Contro A, Zamboni GA, De Robertis R. Retrograde percutaneous transjejunal creation of biliary neoanastomoses in patients with complete hepaticojejunostomy dehiscence. J Vasc Interv Radiol 2015;26:1544-1549.

2. Righi D, Franchello A, Ricchiuti A, Breatta AD, Versace K, Calvo A, et al. Safety and efficacy of the percutaneous treatment of bile leaks in hepaticojejunostomy or split-liver transplantation without dilatation of the biliary tree. Liver Transpl 2008;14:611-615.

3. May K, Hunold P. Leakage of hepaticojejunal anastomosis: radiological interventional therapy. Visc Med 2017;33:192-196.

4. Mauri G, Mattiuz C, Sconfienza LM, Pedicini V, Poretti D, Melchiorre $\mathrm{F}$, et al. Role of interventional radiology in the management of complications after pancreatic surgery: a pictorial review. Insights Imaging 2015;6:231-239.

5. Gupta P, Gupta J, Kumar-M P. Imaging in obstructive jaundice: what a radiologist needs to know before doing a percutaneous transhepatic biliary drainage. J Clin Interv Radiol ISVIR 2020;4:31-37.

6. Fontein DB, Gibson RN, Collier NA, Tse GT, Wang LL, Speer TG, et al. Two decades of percutaneous transjejunal biliary intervention for benign biliary disease: a review of the intervention nature and complications. Insights Imaging 2011;2:557-565.

7. Koch M, Garden OJ, Padbury R, Rahbari NN, Adam R, Capussotti L, et al. Bile leakage after hepatobiliary and pancreatic surgery: a definition and grading of severity by the International Study Group of Liver Surgery. Surgery 2011;149:680-688.

8. Rossi UG, Cariati M. Intrahepatic biliary ductal dilatation. N Engl J Med 2020;382:364.

9. Yoon YI, Hwang S, Ko GY, Lee JJ, Kang CM, Seo JH, et al. Balloon dilation of jejunal afferent loop functional stenosis following left hepatectomy and hepaticojejunostomy long time after pylorus-preserving pancreaticoduodenectomy: a case report. Korean J Hepatobiliary Pancreat Surg 2015;19:66-70.

10. Rossi UG, Passoni GR, Rigamonti P, Cariati M. Percutaneous transhepatic biliojejunal rendezvous technique for biliary obstruction with Billroth II anatomy. Endoscopy 2014;46 Suppl 1 UCTN:E293-E294.

11. Mansueto G, Gatti FL, Boninsegna E, Conci S, Guglielmi A, Contro A. Biliary leakage after hepatobiliary and pancreatic surgery: a classification system to guide the proper percutaneous treatment. Cardiovasc Intervent Radiol 2020;43:302-310.

12. Kaya B, Ozcabi Y, Tasdelen I, Onur E, Memisoglu K. Nasogastric tube placement into the hepaticojejunostomy anastomosis in pancreaticoduodenectomy: a simple surgical technique for prevention of bile leak. Korean J Hepatobiliary Pancreat Surg 2016;20:89-92.

13. Rossi UG, Seitun S, Ferro C. Brilliant green emerald-like bile. J Vasc Interv Radiol 2012;23:1359. 